\title{
Uso de prebióticos à base de mananoligossacarídeo em rações para frangos de corte
}

\author{
Luiz Fernando Teixeira Albino', Fábio Antônio Feres ${ }^{2}$, Marli Arena Dionizio ${ }^{3}$, Horacio Santiago \\ Rostagno ${ }^{1}$, José Geraldo de Vargas Júnior ${ }^{4}$, Débora Cristine de Oliveira Carvalho ${ }^{3}$, Paulo \\ Cezar Gomes ${ }^{1}$, Carlos Henrique Rocha Costa ${ }^{5}$
}

\footnotetext{
${ }^{1}$ Departamento de Zootecnia da Universidade Federal de Viçosa.

${ }^{2}$ Mestre em Zootecnia - UFV.

3 Pós-graduação em Zootecnia da Universidade Federal de Viçosa.

${ }^{4}$ Departamento de Zootecnia - UFES.

${ }^{5}$ Bolsista de Iniciação Científica da FAPEMIG.
}

RESUMO - O experimento foi realizado com objetivo de estudar o uso de mananoligossacarídeos (standard e concentrado) em rações para frangos de corte. Foram utilizados 1.080 pintos de corte machos da marca Ross, com peso médio inicial de $42 \mathrm{~g}$, distribuídos em um delineamento inteiramente ao acaso, com seis tratamentos e nove repetições de 20 aves por unidade experimental. As aves foram criadas em cama de maravalha reaproveitada, utilizada anteriormente por outro lote. Foram testados os tratamentos: testemunha, antibiótico (avilamicina), mananoligossacarídeo alta concentração (MOS AT), mananoligossacarídeo standard (MOS ST), MOS AT + avilamicina e MOS ST + avilamicina para avaliação do ganho de peso, do consumo de ração, da conversão alimentar, da viabilidade e do rendimento de cortes. No período de 1 a 21 dias de idade, não foram encontradas diferenças significativas entre os tratamentos para as variáveis consumo de ração, conversão alimentar e viabilidade econômica. Os resultados de desempenho das aves alimentadas com ração contendo MOS ST ou MOS AT, combinados ou não com o antibiótico, não diferiram dos obtidos nos tratamentos com avilamicina e da ração basal. No período de 1 a 42 dias de idade, a adição de MOS ST, combinado ou não com avilamicina, melhorou o ganho de peso das aves, enquanto o MOS AT afetou de forma negativa o ganho de peso. A conversão alimentar, o consumo de ração e a viabilidade econômica não foram influenciados pelos tratamentos. No entanto, o uso de aditivos em rações para frango de corte melhorou o rendimento de cortes e reduziu a gordura abdominal.

Palavras-chave: aditivo, antibiótico, eficiência alimentar, MOS

\section{Use of mannaoligosaccharid based prebiotic in the broiler diets}

\begin{abstract}
This trial was conducted to study the use of mannanoligosaccharide (standard and concentrate) on chicks diets. One thousand and eigthy 1-d male chicks (Ross) averaging $42 \mathrm{~g}$ of initial BW were assigned to a completely randomized experiment with nine replications of 20 birds per experimental unit. The following treatments were analyzed: control, antibiotic (avilamicine), mannaoligosaccharide high concentration (MOS AT), mannaoligosaccharide standard (MOS ST), MOS AT + antibiotic, and MOS ST + antibiotic. The birds were reared in wood powder, reused of a lot previously housed. Weight gain, feed intake, feed:gain ratio, viability and prime cuts yield were evaluated. In the period from 1 to 21 days old, no significant differences across the treatments on feed intake, feed:gain ratio, and viability were found. The results of performance of birds fed diet containing MOS ST or MOS AT combined or not with the antibiotic, did not differ form those of treatments with avilamicin and control. In the period from 1 to 42 days old, mannanoligosaccharides standard (MOS ST) combined or not with avilamicin, increased weight gain, whereas the mannaoligosaccharide high concentration (MOS AT) showed did not affect weight gain. Feed:gain ratio, feed intake and viability were not affected by the different treatments, but the additives increased prime cuts yield and it decreased abdominal fat.
\end{abstract}

Key Words: additive, antibiotic, feed efficiency, MOS

\section{Introdução}

A escolha de um bom promotor de crescimento deve basear-se em dois fatores: aspecto econômico e segurança. É inquestionável que a relação custo:benefício favorece o uso de antibióticos como aditivo. Todavia, a segurança alimentar começou a ser questionada, principalmente em razão do uso rotineiro desse aditivo na alimentação animal.

O uso de drogas estratégicas para a saúde humana tem sido limitado na Europa por favorecer o desenvolvimento 
de resistência bacteriana, restringindo significativamente os tipos de antibióticos permitidos na produção animal (Swick, 1996).

A avilamicina é um dos antibióticos mais utilizados no Brasil, por seu uso ainda ser permitido pelo Ministério da Agricultura. Entretanto, há possibilidade de os microrganismos adquirirem resistência ao antibiótico com sua adição contínua em dose subterapêutica nas dietas das aves. Yoshimura et al. (2000) observaram que os antibióticos avilamicina e virginiamicina, quando usados como promotores de crescimento em rações para frangos de corte, provocaram o aparecimento de culturas de Enterococcus faecium com 12,4 e 27,4\% de resistência, respectivamente.

$\mathrm{O}$ aparecimento de microrganismos resistentes a um antibiótico aumenta conforme a intensidade de seu uso. Em estudos com frangos de corte na Dinamarca, Aarestrup et al. (2000) avaliaram, em 18 aviários, a resistência do microrganismo Enterococcus faecium à avilamicina. Em todos os oito aviários nos quais se utilizou o antibiótico durante um ano, registrou-se a presença de microrganismos resistentes e, entre os dez aviários nos quais não se utilizou avilamicina durante dois anos, somente em três não foram encontrados Enterococcus faecium resistentes.

Atualmente, diversas organizações têm se manifestado contra o uso desse aditivo em rações avícolas. Setores da imprensa, órgão ligados à saúde, ONGs, entre outros, estão sensibilizando a opinião pública, principalmente em países desenvolvidos, como os europeus, quanto aos possíveis problemas da adição de antibióticos nas rações, estimulando restrições por parte do mercado consumidor de carne e ovos. Assim, surge em muitos países a regulamentação dos aditivos alimentares, com indicação e controle de dosagens e produtos específicos.

Nos últimos anos, tem sido crescente o interesse pelo uso de prebióticos como alternativa para esse problema, pois seu uso poderia eliminar problemas como resistência bacteriana e resíduos de antibióticos nos produtos avícolas, além de melhorar a imagem dos produtos avícolas perante o mercado consumidor.

Gibson \& Roberfroid (1995) descrevem algumas características desejáveis de um prebiótico: não ser metabolizado ou absorvido durante a passagem pelo trato digestivo superior; deve servir de substrato para as bactérias intestinais benéficas (que serão estimuladas a crescer e/ou tornar-se metabolicamente ativas); possuir capacidade de alterar a microbiota intestinal de forma benéfica ao hospedeiro; e induzir efeitos benéficos sistêmicos ou apenas no intestino do hospedeiro. Segundo Young (1998), não há dúvidas de que os prebióticos atuam nutrindo e, conseqüentemente, favorecendo as bactérias probióticas que atuarão beneficiando o hospedeiro.
Um dos oligossacarídeos mais pesquisados e de ação prebiótica são os mananoligossacarídeos (MOS), derivados da parede celular de leveduras Saccharomyces cerevisiae. Segundo Spring et al. (2000), a parede celular é separada do conteúdo intracelular e o líquido contendo MOS é evaporado à baixa temperatura (spray dry) para evitar a destruição da parte funcional da molécula de MOS. A parede celular da levedura é formada por glucanos e mananos, em proporções similares, e pode conter proteínas, enquanto a quitina está presente em pequena quantidade (aproximadamente $1 \%$ ). A estrutura da parede celular da levedura é resistente à degradação das enzimas e bactérias do aparelho digestivo.

A resistência à digestão no trato gastrointestinal superior e à fermentação no intestino grosso, segundo Roberfroid \& Slavin (2000), é um dos principais critérios para escolha dos oligossacarídeos como prebióticos. Segundo Shane (2001), os benefícios dos mananoligossacarídeos (MOS) baseiam-se nas propriedades específicas, que incluem a modificação da flora intestinal, a redução da taxa de renovação da mucosa intestinal (turnover) e a estimulação do sistema imune. As glicomananas, em condições de $\mathrm{pH}$ do aparelho digestivo, são capazes de se ligar seletivamente e inativar as micotoxinas no lúmen intestinal. Estas propriedades têm grande potencial para melhorar o desempenho e diminuir a mortalidade de frangos de corte e perus.

Spring et al. (2000) submeteram frangos de corte ao desafio com dois tipos de Salmonela e encontraram menores concentrações destas bactérias no trato intestinal de aves suplementadas com MOS em relação ao tratamento controle.

Macdonald (1995), citado por Swick (1996), em experimento com frangos de corte, observou melhora significativa da conversão alimentar e redução da mortalidade de animais alimentados com MOS. Em trabalho realizado com perus, Parks et al. (2001) observaram aumento no ganho de peso (na $20^{\text {a }}$ semana de vida) e melhora na conversão alimentar (de 0 a 3 semanas e de 15 a 18 semanas de idade) dos animais que receberam MOS em relação ao tratamento controle. Os autores concluíram que o MOS pode ser utilizado como alternativa aos antibióticos.

Os objetivos neste trabalho foram estudar o rendimento de cortes e o desempenho de frangos de corte alimentados com dietas contendo mananoligossacarídeos e avaliar a viabilidade de substituição dos antibióticos, sem que ocorra perda dos atuais índices de produtividade alcançados.

\section{Material e Métodos}

O experimento foi realizado no setor de avicultura do Departamento de Zootecnia da Universidade Federal de Viçosa no período de outubro a novembro de 2001. Foram 
utilizados 1.080 pintos de corte machos, da marca comercial Ross de 1 a 42 dias de idade, distribuídos em boxes (1,1 x 2,0 m) alojados em galpão de alvenaria telado e coberto com telhas de amianto. O manejo geral foi de acordo com o manual da marca comercial. As aves foram alojadas em cama de maravalha reaproveitada, utilizada anteriormente por outro lote, com o objetivo de proporcionar desafio sanitário. As rações experimentais utilizadas foram formuladas à base de milho e farelo de soja, de forma a atender às recomendações nutricionais (Tabela 1), segundo Rostagno et al. (2000). Os produtos foram adicionados em substituição ao material inerte (areia lavada). Todas as aves receberam ração e água à vontade.

As aves, com peso médio inicial de $42 \mathrm{~g}$, foram distribuídas em um delineamento inteiramente ao acaso, com seis tratamentos (Tabela 2), nove repetições e 20 aves por unidade experimental. Foram utilizados três tipos de aditivos: um promotor de crescimento (avilamicina) e dois prebióticos (mananoligossacarídeos) com diferentes concentrações de leveduras, denominados MOS standard e MOS alta concentração, incluídos nas dietas na forma isolada ou associados.

Foram avaliados o ganho de peso, o consumo de ração, a conversão alimentar, o rendimento de carcaça e dos cortes, a viabilidade e o fator de produção.

Aos 42 dias de idade, após a pesagem das aves, foram selecionados três animais representantes do peso médio da unidade experimental, para avaliação do rendimento de carcaça, perna (coxa + sobrecoxa), peito (com pele e osso), filé de peito e gordura abdominal. O rendimento de carcaça,

Tabela 1 - Composição das rações utilizadas nos períodos de 1 a 21 e de 22 a 42 dias de idade

Table 1 - Ingredient composition of the basal diets (1-21 and 22-42 days of age)

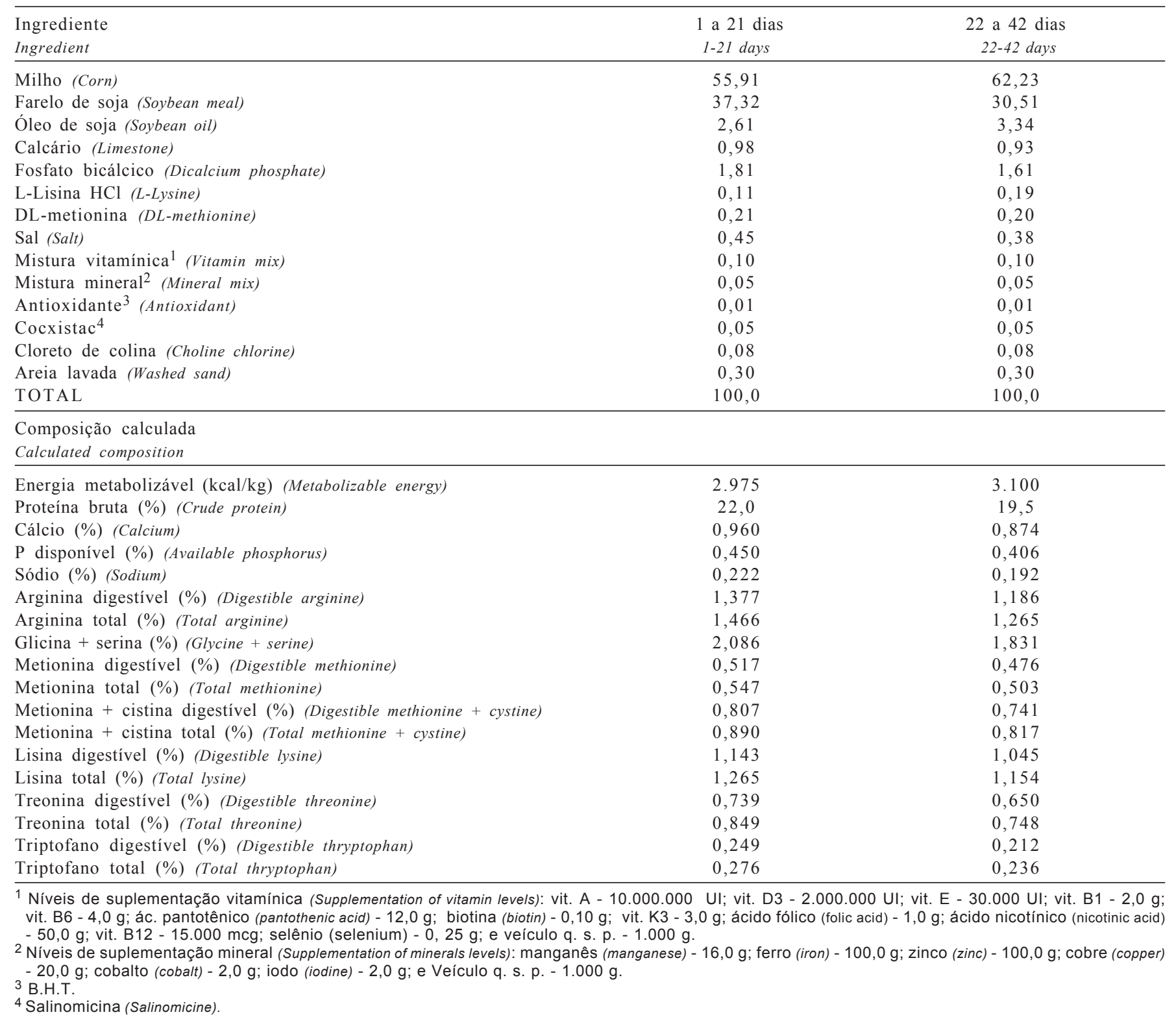


Tabela 2 - Tratamentos utilizados no experimento nos períodos de 1 a 21 e de 22 a 42 dias de idade Table 2 - Experimental treatments from 1-21 and 22-42 days of age

\begin{tabular}{|c|c|}
\hline \multirow{2}{*}{$1-$} & Controle (ração basal) \\
\hline & Control (basal diet) \\
\hline $2-$ & Tratamento $1+$ avilamicina $^{1}$ \\
\hline & Treatment $1+$ avilamicine $^{1}$ \\
\hline $3-$ & $\begin{array}{l}\text { Tratamento } 1+2 \mathrm{~kg} / \mathrm{t} \text { de } \operatorname{MOS}^{2} \text { alta concentração } \\
\text { Treatment } 1+2 \mathrm{~kg} / \mathrm{t} \text { of MOS high concentration }\end{array}$ \\
\hline $4-$ & $\begin{array}{l}\text { Tratamento } 1+2 \mathrm{~kg} / \mathrm{t} \text { de MOS standard } \\
\text { Treatment } 1+2 \mathrm{~kg} / \mathrm{t} \text { of MOS standard }\end{array}$ \\
\hline $5-$ & $\begin{array}{l}\text { Tratamento } 1+\text { avilamicina }^{1}+2 \mathrm{~kg} / \mathrm{t} \text { de MOS alta concentração } \\
\text { Treatment } 1+\text { avilamicine }^{1}+2 \mathrm{~kg} / \mathrm{t} \text { of } \mathrm{MOS} \text { high concentration }\end{array}$ \\
\hline $6-$ & $\begin{array}{l}\text { Tratamento } 1+\text { avilamicina }^{1}+2 \mathrm{~kg} / \mathrm{t} \text { de MOS standard } \\
\text { Treatment } 1+\text { avilamicine }^{1}+2 \mathrm{~kg} / \mathrm{t} \text { of } \text { MOS standard }\end{array}$ \\
\hline
\end{tabular}

17 ppm de 1 a 21 dias e 5 ppm de 22 a 42 dias de idade usando Surmax 100 ( $10 \%$ de avilamicina).

2 MOS - mananoligossacarídeo.

17 ppm from 1-21 days and 5 ppm from 22-42 days of age using Surmax 100 (10\% of avilamicine).

${ }^{2}$ MOS-mannaoligosaccharide.

em porcentagem, foi obtido pela relação entre o peso da carcaça eviscerada (sem os pés, cabeça e pescoço) e o peso vivo. Os rendimentos de perna, peito, filé de peito e gordura abdominal, por sua vez, foram estimados considerando-se a carcaça eviscerada.

As análises estatísticas foram feitas utilizando-se o programa SAEG - Sistema de Análises Estatísticas e Genéticas - UFV (1999), por meio de uma análise de variância e do teste de média Student Newman Keuls.

\section{Resultados e Discussão}

No período de 1 a 21 dias de idade, a adição de avilamicina à ração basal melhorou o ganho de peso, sem afetar o consumo de ração e a conversão alimentar, indicando que o desafio sanitário natural com cama de um lote permitiu a manifestação das respostas, que poderiam ser diferentes caso outros níveis de aditivos fossem utilizados simulta- neamente ao desafio, como relatado por Corrêa et al. (2000), que observaram que o desempenho dos animais dos tratamentos com dois tipos de probióticos e com um antibiótico não diferiu do obtido no tratamento sem aditivo. Com a adição do MOS standard (MOS ST) ou o MOS alta concentração (MOS AT), combinados ou não com avilamicina, os efeitos sobre o ganho de peso não diferiram dos tratamentos com ou sem avilamicina na ração basal. Entretanto, para o consumo de ração e a conversão alimentar, não foram encontradas diferenças significativas $(P>0,05)$ (Tabela 3). Esses resultados são semelhantes a outros descritos na literatura (Pelicano et al., 2004, 2003; Sato et al., 2002; Vargas et al., 2000).

No período de 22 a 42 dias de idade, a adição de MOS $\mathrm{AT}+$ avilamicina à ração basal não afetou de forma significativa o ganho de peso das aves. Todavia, tanto os tratamentos com MOS ST, MOS AT e MOS ST + avilamicina como o tratamento avilamicina melhoraram de forma significativa o ganho de peso. Ressalta-se que, no período de 22

Tabela 3 - Desempenho de pintos de corte machos submetidos aos tratamentos com prebióticos e antibióticos e suas combinações no período de 1 a 21 dias

Table 3 - Performance of male broiler chicks from 1-21 days submitted to prebiotic and antibiotic treatments and its combinations

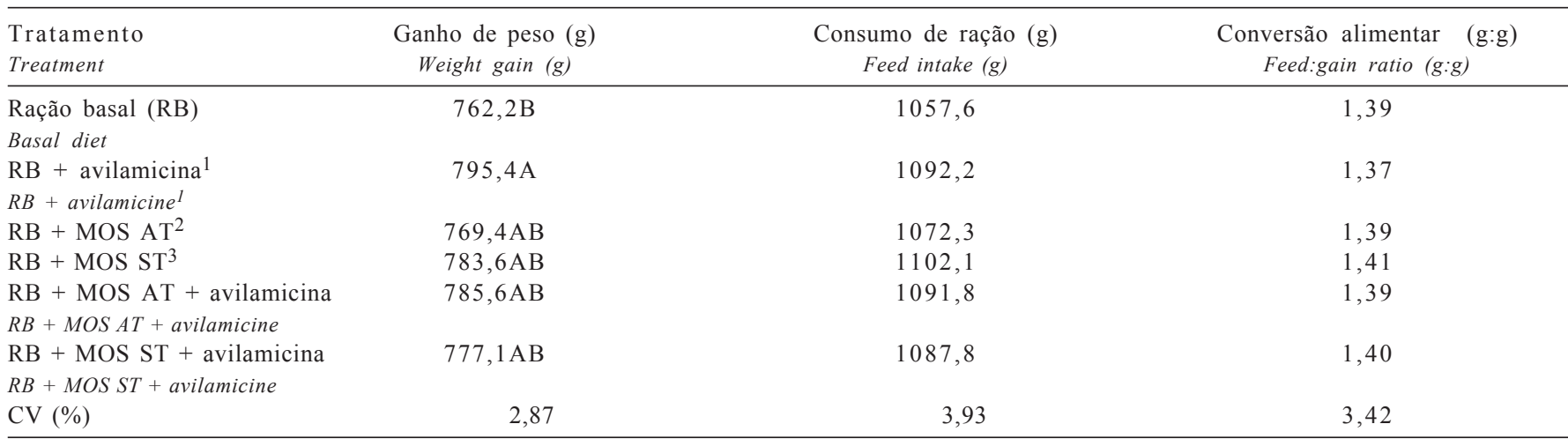

Médias seguidas pela mesma letra na coluna não diferem a $5 \%$ de probabilidade pelo teste Student Newman Keuls

Means followed by the same letter in the column do not differ at $5 \%$ of probability, Student Newman Keuls test.

17 ppm.

2 mananoligossacarídeo alta concentração - $2 \mathrm{~kg} / \mathrm{t}$ (mannaoligosaccharide high concentration - $2 \mathrm{~kg} / \mathrm{t}$ ).

3 mananoligossacarídeo standard - $2 \mathrm{~kg} / \mathrm{t}$ (mannaoligosaccharide standard - $2 \mathrm{~kg} / \mathrm{t}$ ). 
a 42 dias de idade das aves, houve também o efeito residual dos tratamentos a que as aves foram submetidas no período inicial, de 1 a 21 dias de idade. Independentemente do aditivo utilizado, não houve efeito sobre o consumo e a conversão alimentar (Tabela 4).

De forma semelhante, Hofacre et al. (2003), trabalhando com prebióticos, não notaram diferenças significativas para ganho de peso entre os tratamentos, mas verificaram para conversão alimentar. As aves que receberam a combinação de mananoligossacarídeos e ácido lático apresentaram conversão alimentar similar à observada nos tratamentos com antibiótico e controle.

A adição de avilamicina e do mananoligossacarídeo standard, isoladamente ou em combinação, proporcionou efeitos benéficos no ganho de peso das aves no período de 1 a 42 dias de idade (Tabela 5). Estes resultados corroboram os obtidos por Parks et al. (2001), que, em estudo com perus, testaram diferentes antibióticos e MOS e observaram que estes tratamentos aumentaram o ganho de peso dos animais. Resultados semelhantes também foram relatados por Rostagno et al. (2003), que, avaliando o efeito de prebiótico à base de MOS em rações contendo milhos de diferentes qualidades nutricionais, verificaram melhoria no ganho de peso, na conversão alimentar e no fator de produção das aves.

Contrariando os resultados deste trabalho, Waldroup et al. (2003a), trabalhando com antibiótico, adição de Bio$\operatorname{MOS}^{\circledR}$ ou a combinação de antibiótico e Bio-MOS ${ }^{\circledR}$, verificaram que o peso corporal dos frangos não sofreu influência significativa dos tratamentos. A conversão alimentar ( $\mathrm{kg}$ de alimento:kg de ganho) aos 42 dias de idade aumentou significativamente tanto com o antibiótico quanto com a adição de Bio-MOS ${ }^{\circledR}$. A conversão alimentar das aves alimentadas com a combinação de antibiótico e Bio-MOS ${ }^{\circledR}$ não foi diferente do tratamento controle. Aos 56 dias de idade, a conversão alimentar das aves submetidas aos tratamentos com antibiótico e com a combinação antibiótico e Bio-MOS ${ }^{\circledR}$ melhorou em relação à do tratamento controle $(\mathrm{P}<0,01)$. Não houve efeito significativo dos tratamentos na mortalidade e no rendimento de carcaça ou de cortes.

Os efeitos sobre o desempenho e o rendimento de carcaça com a utilização de MOS em dietas para frangos de corte são variáveis e podem ser influenciados pelo nível de inclusão na ração, conforme relatado por Waldroup et al. (2003b), Kumprecht \& Zobac (1997), Iji et al. (2001), Eren et al. (1999), Shafey et al. (2001), Collet (2000) e Macari \& Maiorka (2000).

Considerando os resultados obtidos no período total (1-42 dias de idade), observa-se que as aves submetidas ao tratamento com MOS AT na forma isolada apresentaram ganho de peso estatisticamente igual ao obtido nos tratamentos com adição ou não de avilamicina à ração basal. Porém, quando o MOS AT foi combinado com a avilamicina, o ganho de peso não diferenciou do tratamento com ração basal. Quanto aos tratamentos com o MOS ST, combinado ou não com o antibiótico, foram obtidos resultados estatisticamente iguais ao do tratamento com antibiótico na forma isolada, evidenciando que, nessas condições experimentais, o MOS ST pode substituir a avilamicina mantendo o mesmo ganho de peso dos animais. Independentemente do uso dos aditivos nos períodos de 22 a 42 ou de 1 a 42 dias de idade, o consumo de ração e a conversão alimentar não foram significativamente afetados (Tabelas 4 e 5).

A adição de avilamicina ou de MOS ST, combinado ou não à avilamicina na ração basal, melhorou significativa-

Tabela 4 - Desempenho de frangos de corte machos de 22 a 42 dias de idade submetidos a prebióticos, antibiótico e suas combinações na ração

Table 4 - $\quad$ Performance of male broiler chicks from 22-42 days submitted to prebiotic, antibiotic and its combinations

\begin{tabular}{|c|c|c|c|}
\hline $\begin{array}{l}\text { Tratamento } \\
\text { Treatment }\end{array}$ & $\begin{array}{l}\text { Ganho de peso }(\mathrm{g}) \\
\text { Weight gain }(\mathrm{g})\end{array}$ & $\begin{array}{c}\text { Consumo de ração }(\mathrm{g}) \\
\text { Feed intake }(\mathrm{g})\end{array}$ & $\begin{array}{c}\text { Conversão alimentar }(\mathrm{g}: \mathrm{g}) \\
\text { Feed:gain ratio }(\mathrm{g}: \mathrm{g})\end{array}$ \\
\hline Ração basal (RB) & $1645,0 \mathrm{~B}$ & 3509,7 & 2,13 \\
\hline $\begin{array}{l}\text { Basal diet } \\
\mathrm{RB}+\text { avilamicina }^{1}\end{array}$ & $1720,4 \mathrm{~A}$ & 3583,6 & 2,08 \\
\hline $\mathrm{RB}+\operatorname{MOS} \mathrm{AT}^{2}$ & $1693,3 \mathrm{~A}$ & 3553,0 & 2,10 \\
\hline $\mathrm{RB}+\operatorname{MOS} \mathrm{ST}^{3}$ & $1715,7 \mathrm{~A}$ & 3588,8 & 2,09 \\
\hline $\begin{array}{l}\mathrm{RB}+\operatorname{MOS} \mathrm{AT}+\text { avilamicina } \\
R B+\operatorname{MOS} A T+\text { avilamicine }\end{array}$ & $1646,2 \mathrm{~B}$ & 3505,4 & 2,13 \\
\hline $\begin{array}{l}\mathrm{RB}+\mathrm{MOS} \mathrm{ST}+\text { avilamicina } \\
R B+\text { MOS ST + avilamicine }\end{array}$ & $1707,1 \mathrm{~A}$ & 3529,2 & 2,07 \\
\hline CV (\%) & 2,13 & 5,37 & 5,01 \\
\hline
\end{tabular}

Médias seguidas pela mesma letra na coluna não diferem a $5 \%$ de probabilidade pelo teste Student Newman Keuls.

Means followed by the same letter in the column do not differ at $5 \%$ of probability, Student Newman Keuls test.

$15 \mathrm{ppm}$.

2 mananoligossacarídeo alta concentração - $2 \mathrm{~kg} / \mathrm{t}$ (mannaoligosaccharide high concentration - $2 \mathrm{~kg} / \mathrm{t}$ ).

3 mananoligossacarídeo standard - $2 \mathrm{~kg} / \mathrm{t}$ (mannaoligosaccharide standard $-2 \mathrm{~kg} / \mathrm{t}$ ). 
mente $(\mathrm{P}<0,05)$ o peso final das aves (Tabela 6). Este resultado diverge do encontrado por Dionizio et al. (2002), que, em experimento com frangos de até 42 dias de idade, não verificaram diferença no peso final das aves entre os tratamentos controle e com prebiótico e antibiótico.

O uso de avilamicina, MOS ST ou AT, combinados ou não com avilamicina, melhorou os rendimentos de peito, filé de peito e gordura abdominal, contrariando os encontrados por Vargas Jr. et al. (2002), que, utilizando antibiótico, dois tipos de probióticos, prebiótico e suas combinações, não obtiveram diferenças significativas nos cortes comerciais em nenhum dos tratamentos utilizados, possivelmente por não terem submetido as aves a desafio suficiente que justificasse a utilização dos aditivos. Os melhores resultados de rendimento de perna foram obtidos com MOS alta concentração + avilamicina, enquanto os rendimentos de carcaça não foram afetados pelo uso dos aditivos estudados.

Os tratamentos com MOS standard ou alta concentração, combinados ou não com avilamicina, melhoraram o ganho de peso, o rendimento de cortes, a gordura abdominal, o fator de produção e o peso final das aves (Tabelas 7 e 8 ).

Tabela 5 - Desempenho, viabilidade (VI) e fator de produção (FP) de frangos de corte machos de 1 a 42 dias submetidos a tratamentos com prebióticos, antibiótico e suas combinações

Table 5 - Performance, viability (VI) and production factor (PP) of male broiler chicks from 1-42 days submitted to prebiotic, antibiotic and its combinations

\begin{tabular}{|c|c|c|c|c|c|}
\hline $\begin{array}{l}\text { Tratamento } \\
\text { Treatment }\end{array}$ & $\begin{array}{l}\text { Ganho de peso }(\mathrm{g}) \\
\text { Weight gain }(\mathrm{g})\end{array}$ & $\begin{array}{c}\text { Consumo de ração }(\mathrm{g}) \\
\text { Feed intake }(\mathrm{g})\end{array}$ & $\begin{array}{c}\text { Conversão alimentar (g:g) } \\
\text { Feed:gain ratio }(g: g)\end{array}$ & VI $(\%)$ & FP \\
\hline Ração basal (RB) & $2416,6 \mathrm{~B}$ & 4567,3 & 1,89 & 90,6 & 276,0 \\
\hline Basal diet & & & & & \\
\hline $\begin{array}{l}\mathrm{RB}+\text { avilamicina }^{1} \\
R B+\text { avilamicine }\end{array}$ & $2515,8 \mathrm{~A}$ & 4675,8 & 1,86 & 92,2 & 297,0 \\
\hline $\mathrm{RB}+\operatorname{MOS} \mathrm{AT}^{2}$ & $2459,1 \mathrm{AB}$ & 4625,3 & 1,88 & 96,1 & 299,4 \\
\hline $\mathrm{RB}+\operatorname{MOS} \mathrm{ST}^{3}$ & $2494,9 \mathrm{~A}$ & 4691,0 & 1,88 & 96,1 & 303,7 \\
\hline $\begin{array}{l}\mathrm{RB}+\operatorname{MOS} \mathrm{AT}+\text { avilamicina } \\
R B+\text { MOS AT }+ \text { avilamicine }\end{array}$ & $2429,0 \mathrm{~B}$ & 4597,2 & 1,89 & 94,4 & 288,4 \\
\hline $\begin{array}{l}\mathrm{RB}+\mathrm{MOS} \mathrm{ST}+\text { avilamicina } \\
R B+\text { MOS ST }+ \text { avilamicine }\end{array}$ & $2483,7 \mathrm{~A}$ & 4617,0 & 1,86 & 92,8 & 295,1 \\
\hline CV $(\%)$ & 1,84 & 4,35 & 4,16 & 7,83 & 7,31 \\
\hline
\end{tabular}

Médias seguidas pela mesma letra na coluna não diferem a $5 \%$ de probabilidade pelo teste Student Newman Keuls.

Means followed by the same letter in the column do not differ at $5 \%$ of probability, Student Newman Keuls test.

17 ppm de 1 a 21 dias e 5 ppm de 22 a 42 dias (7 ppm from 1-21 days and 5 ppm from 22-42 days).

2 mananoligossacarídeo alta concentração - $2 \mathrm{~kg} / \mathrm{t}$ (mannaoligosaccharide high concentration - $2 \mathrm{~kg} / \mathrm{t}$ ).

3 mananoligossacarídeo standard - $2 \mathrm{~kg} / \mathrm{t}$ (mannaoligosaccharide standard $-2 \mathrm{~kg} / \mathrm{t}$ ).

Tabela 6 - Peso final (PF), rendimentos de carcaça (RC) e de cortes comerciais de frangos de corte machos de 1 a 42 dias submetidos a tratamentos com prebióticos, antibiótico e suas combinações

Table 6 - Final weight (PF), yields of carcass (RC) and prime cuts of male broiler chicks from 1-42 days submitted to prebiotic, antibiotic and its combinations

\begin{tabular}{|c|c|c|c|c|c|c|}
\hline $\begin{array}{l}\text { Tratamento } \\
\text { Treatment }\end{array}$ & $\mathrm{PF}(\mathrm{g})$ & $\mathrm{RC}(\%)$ & $\begin{array}{l}\text { Peito }(\%) \\
\text { Breast }(\%)\end{array}$ & $\begin{array}{c}\text { Filé de peito }(\%) \\
\text { Breast fillet }(\%)\end{array}$ & $\begin{array}{c}\text { Perna }(\%) \\
\text { Legs }(\%)\end{array}$ & $\mathrm{GAB}^{4}(\%)$ \\
\hline $\begin{array}{l}\text { Ração basal (RB) } \\
\text { Basal diet }\end{array}$ & $2454,7 \mathrm{~B}$ & 67,5 & $33,0 \mathrm{C}$ & $24,0 \mathrm{~B}$ & $31,8 \mathrm{~B}$ & $1,71 \mathrm{~A}$ \\
\hline $\begin{array}{l}\mathrm{RB}+\text { avilamicina }^{1} \\
R B+\text { avilamicine }^{I}\end{array}$ & $2553,9 \mathrm{~A}$ & 67,6 & $34,3 \mathrm{~A}$ & $25,4 \mathrm{~A}$ & $32,0 \mathrm{~B}$ & $1,38 \mathrm{~B}$ \\
\hline $\mathrm{RB}+\operatorname{MOS} \mathrm{AT}^{2}$ & $2461,7 \mathrm{~B}$ & 67,6 & $33,8 \mathrm{AB}$ & $24,9 \mathrm{~A}$ & $32,3 \mathrm{~B}$ & $1,53 \mathrm{AB}$ \\
\hline $\mathrm{RB}+\operatorname{MOS} \mathrm{ST}^{3}$ & $2533,1 \mathrm{~A}$ & 67,3 & $33,8 \mathrm{AB}$ & $25,1 \mathrm{~A}$ & $32,3 \mathrm{~B}$ & $1,31 \mathrm{~B}$ \\
\hline $\begin{array}{l}\mathrm{RB}+\operatorname{MOS} \mathrm{AT}+\text { avilamicina } \\
R B+\operatorname{MOS} A T+\text { avilamicine }\end{array}$ & $2467,1 \mathrm{~B}$ & 67,3 & $33,4 \mathrm{~B}$ & $24,7 \mathrm{~A}$ & $32,9 \mathrm{~A}$ & $1,36 \mathrm{~B}$ \\
\hline $\begin{array}{l}\mathrm{RB}+\mathrm{MOS} \mathrm{ST}+\text { avilamicina } \\
R B+M O S S T+\text { avilamicine }\end{array}$ & $2521,8 \mathrm{~A}$ & 67,3 & $34,0 \mathrm{AB}$ & $25,4 \mathrm{~A}$ & $32,4 \mathrm{~B}$ & $1,38 \mathrm{~B}$ \\
\hline CV $(\%)$ & 2,21 & 2,10 & 1,35 & 2,69 & 1,52 & 15,82 \\
\hline
\end{tabular}

Médias seguidas pela mesma letra na coluna não diferem a $5 \%$ de probabilidade pelo teste Student Newman Keuls.

Means followed by the same letter in the column do not differ at $5 \%$ of probability, Student Newman Keuls test.

$17 \mathrm{ppm}$ de 1 a 21 dias e 5 ppm de 22 a 42 dias (7 ppm from 1-21 days and 5 ppm from 22-42 days).

2 mananoligossacarídeo alta concentração - $2 \mathrm{~kg} / \mathrm{t}$ (mannaoligosaccharide high concentration - $2 \mathrm{~kg} / \mathrm{t}$ ).

3 mananoligossacarídeo standard - $2 \mathrm{~kg} / \mathrm{t}$ (mannaoligosaccharide standard - $2 \mathrm{~kg} / \mathrm{t}$ ).

${ }^{4}$ Gordura abdominal (Abdominal fat). 
Tabela 7 - Índice de ganho de peso de aves submetidas a prebióticos, antibiótico e suas combinações na ração basal Table 7 - Index of birds weight gain submitted to prebiotic, antibiotic and its combinations in relation to the basal diet

\begin{tabular}{|c|c|c|c|}
\hline $\begin{array}{l}\text { Tratamento } \\
\text { Treatment }\end{array}$ & $\begin{array}{l}1-21 \text { dias } \\
1-21 \text { days }\end{array}$ & $\begin{array}{l}22-42 \text { dias } \\
22-42 \text { days }\end{array}$ & $\begin{array}{l}1-42 \text { dias } \\
1-42 \text { days }\end{array}$ \\
\hline Ração basal (RB) & 100,0 & 100,0 & 100,0 \\
\hline \multicolumn{4}{|l|}{$\begin{array}{l}\text { Basal diet } \\
\mathrm{RB}+\text { avilamicina }^{1}\end{array}$} \\
\hline$R B+$ avilamicine & 104,3 & 104,6 & 104,1 \\
\hline $\mathrm{RB}+\operatorname{MOS} \mathrm{AT}^{2}$ & 100,9 & 102,9 & 101,8 \\
\hline $\mathrm{RB}+\operatorname{MOS} \mathrm{ST}^{3}$ & 102,8 & 104,3 & 103,2 \\
\hline $\begin{array}{l}\mathrm{RB}+\operatorname{MOS} \mathrm{ST}+\text { avilamicina } \\
R B+\operatorname{MOS} S T+\text { avilamicine }\end{array}$ & 101,9 & 103,8 & 102,8 \\
\hline
\end{tabular}

Médias seguidas pela mesma letra na coluna não diferem a $5 \%$ de probabilidade pelo teste Student Newman Keuls.

Means followed by the same letter in the column do not differ at $5 \%$ of probability, Student Newman Keuls test.

17 ppm de 1 a 21 dias e 5 ppm de 22 a 42 dias (7 ppm from 1-21 days and 5 ppm from 22-42 days).

2 mananoligossacarídeo alta concentração $-2 \mathrm{~kg} / \mathrm{t}$ (mannaoligosaccharide high concentration - $2 \mathrm{~kg} / \mathrm{t}$ ).

${ }^{3}$ mananoligossacarídeo standard $-2 \mathrm{~kg} / \mathrm{t}$ (mannaoligosaccharide standard $-2 \mathrm{~kg} / \mathrm{t}$ ).

Tabela 8 - Índice de rendimento de cortes comerciais, gordura abdominal (GAB), fator de produção (FP) e peso final (PF) de aves submetidas a tratamentos com prebióticos, antibiótico e suas combinações na ração basal

Table 8 - Index of revenue of commercial parts, abdominal fat (GAB), production factor (PF) and final weight (PF) of birds submitted to prebiotic, antibiotic and its combinations

\begin{tabular}{|c|c|c|c|c|c|c|}
\hline $\begin{array}{l}\text { Tratamento } \\
\text { Treatment }\end{array}$ & $\begin{array}{l}\text { Peito } \\
\text { Breast }\end{array}$ & $\begin{array}{l}\text { Filé de peito } \\
\text { Breast fillet }\end{array}$ & $\begin{array}{c}\text { Perna } \\
\text { Legs }\end{array}$ & GAB & FP & $\mathrm{PF}$ \\
\hline Ração basal (RB) & 100,0 & 100,0 & 100,0 & 100,0 & 100,0 & 100,0 \\
\hline Basal diet & & & & & & \\
\hline $\begin{array}{l}\mathrm{RB}+\text { avilamicina }^{1} \\
R B+\text { avilamicine }^{I}\end{array}$ & 104,0 & 106,1 & 100,6 & 80,7 & 107,6 & 104,0 \\
\hline $\mathrm{RB}+\mathrm{MOS} \mathrm{AT}^{2}$ & 102,6 & 104,0 & 101,6 & 89,5 & 108,5 & 100,3 \\
\hline $\mathrm{RB}+\operatorname{MOS} \mathrm{ST}^{3}$ & 102,6 & 104,5 & 101,5 & 76,6 & 110,0 & 103,2 \\
\hline $\begin{array}{l}\mathrm{RB}+\operatorname{MOS} \mathrm{AT}+\text { avilamicina } \\
R B+\operatorname{MOS} A T+\text { avilamicine }\end{array}$ & 101,4 & 102,9 & 103,5 & 100,0 & 104,5 & 100,5 \\
\hline $\begin{array}{l}\mathrm{RB}+\mathrm{MOS} \mathrm{ST}+\text { avilamicina } \\
R B+M O S S T+\text { avilamicine }\end{array}$ & 103,0 & 106,1 & 101,9 & 80,7 & 106,9 & 102,7 \\
\hline
\end{tabular}

Médias seguidas pela mesma letra na coluna não diferem a $5 \%$ de probabilidade pelo teste Student Newman Keuls.

Means followed by the same letter in the column do not differ at $5 \%$ of probability, Student Newman Keuls test.

17 ppm de 1 a 21 dias e 5 ppm de 22 a 42 dias (7 ppm from 1-21 days and 5 ppm from 22-42 days).

2 mananoligossacarídeo alta concentração - $2 \mathrm{~kg} / \mathrm{t}$ (mannaoligosaccharide high concentration - $2 \mathrm{~kg} / \mathrm{t}$ ).

${ }^{3}$ mananoligossacarídeo standard - $2 \mathrm{~kg} / \mathrm{t}$ (mannaoligosaccharide standard $-2 \mathrm{~kg} / \mathrm{t}$ ).

\section{Conclusões}

O uso de aditivo (promotor de crescimento) nas rações melhorou o desempenho de frangos de corte.

Prebióticos à base de mananoligossacarídeo podem substituir o antibiótico avilamicina em rações para frangos de corte.

\section{Literatura Citada}

AARESTRUP, F.M.; BAGER, F.; ANDERSEN, J.S. Association between the use of avilamycin for growth promotion and the occurrence of resistance among Enterococcus faecium from broilers: epidemiological study and changes over time. Microbial Drug Resistance, v.6, n.1, p.71-75, 2000.

COLLET, S. Nutrição, Imunidade e Produtividade. In: RONDA LATINO-AMERICANA - O FUTURO DA ALIMENTAÇÃO, 10., 2000, Brasil. Palestras... Brasil: Alltech, 200. p.20-30.
CORRÊA, G.S.S.; GOMES, A.V.C.; CORRÊA, A.B. et al. Desempenho de frangos de corte alimentados com diferentes promotores de crescimento. In: REUNIÃO ANUAL DA SOCIEDADE BRASILEIRA DE ZOOTECNIA, 37., 2000, Viçosa, MG. Anais... São Paulo: Gnosis, 2000. CD-ROM. Nutrição de não ruminantes. NNR-0300.

DIONIZIO, M.A.; BERTECHINI, A.G.; TEIXEIRA, A.S. et al. Prebióticos como promotores de crescimento para frangos de corte - desempenho e rendimento de carcaça. In: REUNIÃO ANUAL DA SOCIEDADE BRASILEIRA DE ZOOTECNIA, 39., 2002, Recife. Anais... Recife: Technomédia, 2002. CD-ROM. Nutrição de não-ruminantes. 05sbz1277.

GIBSON, G.R.; ROBERFROID M.B. Dietary modulation of the human colonic microbiota: introducing the concept of prebiotics. Journal of Nutrition, v.125, n.6, p.1401-1412, 1995.

HOFACRE, C.L.; BEACORN, T.; COLLET, S.; MATHIS, G. Using Competitive Exclusion, Mannan-oligosaccharide and Other Intestinal Products to Control Necrotic Enteritis. Journal Applied Poultry Research, n.12, p.60-64, 2003.

IJI, P.A.; SAKI, A.A.; TIVEY, D.R. Intestinal Structure and function of broiler chickens on diets supplemented with a mannan 
oligosaccharide. Journal Science Food Agriculture, v.81, p.1186-1192, 2001.

KUMPRECHT, I.; ZOBAC. The effect of Mannanoligosaccharides in feed mixtures on the performance of broilers. Zivocisna Vyroba, n.42, p.117-124, 1997.

MACARI, M. MAIORKA, A. Função Gastrintestinal e seu impacto no rendimento avícola. In: CONFERÊNCIA APINCO'2000 DE CIÊNCIA E TECNOLOGIA AVÍCOLAS, 2000, Campinas. Anais... Campinas: Fundação Apinco de Ciência e Tecnologia Avícolas, 2000. v.2, p.161-174.

PARKS, C.W.; GRIMES, J.L.; FERKET, P.R. et al. The effect of mannanoligosaccharides, bambermycins, and virginiamycin on performance of large white male market turkeys. Poultry Science, v.80, n.6, p.718-723, 2001

PELICANO, E.R.L.; SOUZA, P.A.; SOUZA, H.B.A. et al. Utilização de probióticos e/ou prebióticos como promotores de crescimento em rações iniciais de frangos de corte. Revista Brasileira de Ciência Avícola, Suplemento 6, p.17, 2004.

ROBERFROID, M.B.; SLAVIN, J. Nondigestible oligosaccharides. Critical Reviews in Food Science Nutrition, v.40, n.60, p.461-480, 2000. (Abstract)

ROSTAGNO, H.S.; ALBINO, L.F.T.; DONZELE, J.L. et al. Tabelas brasileiras para aves e suínos. Composição de alimentos e exigências nutricionais. Viçosa, MG: Universidade Federal de Viçosa, 2000. 141p.

ROSTAGNO, H.S.; ALBINO, L.F.T.; TOLEDO, R.S. et al. Avaliação de prebióticos à base de mananoligossacarídeos em rações de frangos de corte contendo milhos de diferente qualidade nutricional. Revista Brasileira de Ciência Avícola, Suplemento 5, p.52, 2003.

SATO, R.N.; LODDI, M.M.; NAKAGHI, L.S.O. Uso de antibiótico e/ou probiótico como promotores de crescimento em rações iniciais de frangos. Revista Brasileira de Ciência Avícola, Suplemento 4, p.37, 2002.

SHAFEY, T.M., AL-MUFAREJ, S., SHALABY, M.I., JARLENABI, A. J. The Effect of feeding mannanoligosaccharides (Bio-Mos) on the performance of meat chickens under two different vaccination programs. Asian-Australian Journal Animal Science, n. 14, p.559-563, 2001.

SHANE, S.M. Mechanisms and benefits of mannanoligosaccharides in poultry nutrition. SYMPOSIUM ON BIOTECHNOLOGY. 2001. Acesso em: 03 de set. 2001. Disponível em: http://www.zootecnica.it/ nutrition.html.
SPRING, P.; WENK, C.; DAWSON, K.A. et al. The effects of dietary mannaoligosaccharides on cecal parameters and the concentrations of enteric bacteria in the ceca of salmonellachallenged broiler chicks. Poultry Science, v.79, n.2, p.205211,2000

SWICK, R.A. Role of growth promotants in poultry and swine feed. 1996. Acesso em: 09/09/2001. Online. Disponível em: http://www.asasea.com/technical/an04-1996.html.

UNIVERSIDADE FEDERAL DE VICOSA - UFV. SAEG - Sistema de análises estatísticas e genéticas. Versão 8.0.Viçosa, MG: 1999. 59p. (Manual do usuário).

VARGAS JR., J.G.; TOLEDO, R.S.; ALBINO, L.F.T. et al Características de carcaça de frango de corte, submetidos a rações contendo probióticos, prebióticos e antibióticos. In: REUNIÃO ANUAL DA SOCIEDADE BRASILEIRA DE ZOOTECNIA, 39., 2002, Recife. Anais... Recife: Technomédia, 2002. CDROM. Nutrição de não-ruminantes. 05sbz986.

VARGAS JR., J.G.; TOLEDO, R.S.; ALBINO, L.F.T. et al. Uso de probióticos e prebióticos em rações de frangos de corte. Revista Brasileira de Ciência Avícola, Suplemento 2, p.31, 2000.

WALDROUP, P.W.; OVIEDO-RONDON, E.O.; FRITTS, C.A Comparison of Bio-Mos ${ }^{\circledR}$ antiobiotic feeding programs in broiler diets containing cooper sulfate. International Journal of Poultry Science, v.2, n.1, p.28-31, 2003a.

WALDROUP, P.W.; FRITTS, C.A.; FENGLAN, Y. Utilization of Bio-Mos ${ }^{\circledR}$ Mannan Oligosaccharide and Bioplex ${ }^{\circledR}$ copper in broiler diets. International Journal of Poultry Science, v.2, n.1, p.44-52, 2003b.

YOUNG, J. European market developments in prebiotic- and probiotic-containing foodstuffs. British Journal of Nutrition, v.80, n.4, p.231-233, 1998

YOSHIMURA, H.; ISHIMARU, M.; ENDOH, Y.S. et al. Antimicrobial susceptibilities of enterococci isolated from faeces of broiler and layer chickens. Journal of Applied Microbiology, v. 31, n. 6, p. $427-432,2000$ 